\title{
Principle for the Working of the Lithium-Ion Battery
}

\author{
Kai Wai Wong1, Wan Ki Chow ${ }^{2}$ \\ ${ }^{1}$ Department of Physics and Astronomy, University of Kansas, Lawrence, USA \\ ${ }^{2}$ Department of Building Services Engineering, The Hong Kong Polytechnic University, Hong Kong, China \\ Email: kww88ng@gmail.com
}

How to cite this paper: Wong, K.W. and Chow, W.K. (2020) Principle for the Working of the Lithium-Ion Battery. Journal of Modern Physics, 11, 1743-1750. https://doi.org/10.4236/jmp.2020.1111107

Received: September 16, 2020

Accepted: November 2, 2020

Published: November 5, 2020

Copyright ( 2020 by author(s) and Scientific Research Publishing Inc. This work is licensed under the Creative Commons Attribution International License (CC BY 4.0).

http://creativecommons.org/licenses/by/4.0/

\begin{abstract}
The technological advances in Lithium-ion batteries have created many new applications, including electric vehicles. In this short note, we shall explain in simple terms the basic physics why and how it is possible to have high energy capacity in Lithium-ion batteries. However, heating has been a common problem and without appropriate design, they might give fire and explosion as reported.
\end{abstract}

\section{Keywords}

Effective Coulomb Potential for Atomic Orbitals, Bohr Atomic Orbital Energies, Changes Due to Space Dimensions Symmetries, Change in Lithium Metal Ionized Valence Bands Binding

\section{Introduction}

Lithium-ion (Li-ion) batteries [1]-[8] have high specific energy, high efficiency and long service life and have become the power supply in many applications. Billions of units are manufactured per year as electrochemical storage systems in renewable energy plants, power systems for electric vehicles and as power sources for the electronics consumer market including smart phones, tablets, notebooks and laptops.

However, many field incidents occurred for Li-ion battery [9] [10] packs all over the world. Fires involving Li-ion batteries must therefore be studied more thoroughly when widely used. How a Li-ion battery will ignite by itself and what are the consequences under a flashover room fire should be studied. The first step is to understand the physics [11] [12] [13] behind why it is possible to have high energy capacity. 


\section{Physical Principles}

Li has atomic number 3 with 1 electron at principal quantum number $n=2$ and single pair or 2 elections at $n=1,1 \mathrm{~s}$. The atomic structure is similar to hydrogen $H$, except that in $H$, the outermost electron has principal quantum number $n$ $=1$. The electronic binding energy for $H$, from Bohr's model [14] [15] in 3D space symmetry is given by $-R$, where $R$ is the Rydberg constant. For the $\operatorname{Li} n=$ $2,1 \mathrm{~s}$ electron, the electronic binding energy is given by $-R / 4$, because the outermost electron experiences of the Pauli exclusion principle, each electron in the atom observed equally a net $+e$ charge due to the effective screening of the nucleus by the remaining the electron charge $-2 e$ at $n=1$. The total electron binding energy is thus $-2 R-R / 4=-9 R / 4$. Should we ionize $H$, it will require an energy $E=R$. The first ionization energy of $\mathrm{Li}$ is not just $R / 4$. Because when one electron is removed each of the remaining 2 electrons each would observe an effective $+2 \mathrm{e}$ charge, due to charge screening of the nucleus by each of these two electrons, hence its total binding energy becomes $-4 R$.

As compared to $-9 R / 4$, an energy of $7 R / 4$ is needed, instead of just the $R / 4$ for $n=2 \mathrm{~s}$ orbital energy. This extra $3 / 2 R$ amount is then stored up in the Li. But of course, Lithium is a metal having a $3 \mathrm{D}$ cubic crystal lattice periodic b.c. It is due to this lattice symmetry point group, the $n=2 \mathrm{~s}$ orbital becomes the conduction band (CB) electron, while the $2 n=1 \mathrm{~s}$ orbitals formed the filled valence band (VB), with a separating gap $G$ roughly of $R / 4$. In making Li into a battery, we remove the $\mathrm{CB}$ electron, thus making it a voltage source. For most other metals, this voltage is just corresponding to the last electron Coulomb binding, yet for Lithium, it is far greater; namely $3 / 2 R$ greater. This extra amount requires that the VB $2 \mathrm{~s}$ orbitals be suppressed lower thus increasing the band gap by that same amount. In fact, what we have discussed is entirely based on 3D atomic symmetry. In a crystal structure, it is possible to make into a 2D thin film. Such a new 2D structure changes our Bohr model analysis. The $2 \mathrm{D}$ atomic levels are given by $-R /[n-1 / 2]^{2}$, where $n=1,2, \cdots$. That means the outer most $s$ orbital energy is $-4 R / 9$ instead of $-R / 4$ while the lower $2 \mathrm{~s}$ orbital energy increases to $-8 R$. By ionization of the CB electron the VB gap $G$ changes from $8 R$ to $16 R$, producing an extra potential energy of $8 R$.

It is this physics that allows the technological advance of making the Lithium battery in terms of $2 \mathrm{D}$ layers. Of course, with the making of Li layers, its electronic orbitals are not perfect $2 \mathrm{D}$, and the atomic energy gain is not exactly $2 \mathrm{D}$. But it still increases the high energy capacity in the recharged battery. The wattage of the battery is proportional to the number of Li metal layers, while the voltage is layer thickness inverse dependent. The wattage limitation to such $\mathrm{Li}$ battery is completely due to the heat it generates.

The Hamiltonian with crystal lattice periodic boundary condition for the ionized Lithium orbitals is

$$
H=\frac{P_{1}^{2}}{2 m}+\frac{P_{2}^{2}}{2 m}-\frac{3 e^{2}}{r_{1}}-\frac{3 e^{2}}{r_{2}}+\frac{e^{2}}{\left|\overline{r_{1}}-\overline{r_{2}}\right|}
$$


where $H$ is the electrons Hamiltonian operator, $e$ the electron charge and $r$ the orbital radius under the Lithium metal periodic lattice boundary condition. Therefore the eigenvalues are given by the band structure as illustrated in Figure 1.

The repulsive electron-electron potential in effect is responsible for reducing the effective Hamiltonian to the $2 \mathrm{~s}$ states by realizing the Pauli exclusion principle to

$$
H \cong \frac{P^{2}}{2 m}-\frac{Z e^{2}}{r}
$$

where $Z=2$.

The band structure change of Li-ion metal is shown in Figure 1 .

The Li-ion voltage $V$ is given by $E_{f}$ and $G$ as:

$$
V=E_{f}+G+\Delta G
$$

The quantum averaged potential change $\Delta G$ is:

$$
\Delta G=\left\langle\frac{e^{2}}{r}\right\rangle
$$

A schematic band structure for the Lithium metal and its corresponding ionized band structure are shown in Figure 1. The horizontal axis of each band diagram is in momentum unit.

Actually, the similar quantum chemistry approach can be applied to ionized Sodium, $\mathrm{Na}$, which has atomic state $n=3,1 \mathrm{~s}$. This Bohr state changes significantly the effective charges observed by the lower energy level electrons, and hence changes significantly the VB energy level changes, reducing the voltage capacity. However, it also reduces the heating when used as a battery material, but not enough for transportation application.

Perhaps sodium ion is in fact naturally derived from salt and used in bio-systems, such as the light emission in the firefly. Thus the future technological improvement lies in the design in heat insulation and dissipation and not in replacing $\mathrm{Li}$ by Na. Because Li-ion battery powered vehicles produce no emission, it is environmentally clean, compact, rechargeable, as well as maintenance free, making it the future choice replacing the combustion engine for transportation. Recently,

\section{CB}

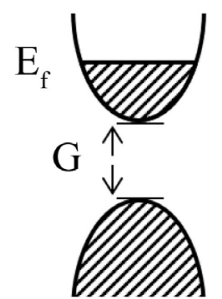

VB

Normal metal

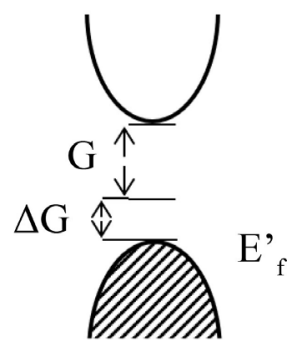

Ionized metal

Figure 1. Band structure change. 
a new Li-ion battery design that substantially increases its power was reported [11]. The problem to tackle the heating problem still remains.

\section{Safety Aspects}

Although Li-ion battery is rapidly adopted [16], safety concerns, both fire and explosion hazards, are threatening widespread adoption. Battery safety can be broadly classified into three categories:

1) Cell design depending on selected materials and circuitry.

Manufacturers are attempting to produce safer [17] products. There might be electric protection circuit.

2) Use of product in scenarios unexpected [18] by the supplier.

Fire and explosion hazards of Li-ion batteries in phones or electric cars in crowded space should be carefully watched [19]. For example, the thermal behavior of cellular phone models which have been complained to be too hot should be studied experimentally. Both smart phones and tablet computers might catch fires accidentally [20] and even explode by themselves. The consequence can be very hazardous in crowded areas allowing phone charging.

3) Accidents coming from normal recommended operating conditions, possibly due to thermal runaway.

Very limited theory is available on explaining thermal runaway. Thermal runaway can be described [10] by the situation when a cell undergoes spontaneous self-destruction under normal operating conditions with two events occurring in sequence:

- High power generation to result in high-temperature hot spots.

- Triggering of the propagation of negative electrode by such local hot spots to increase the temperature of the entire cell, giving further chemical reactions or even combustion.

Flammable gases released would pose fire and explosion hazards for the compartment housing the cells. However, there is little available information characterizing the flammability properties of the gases released after cell thermal runaway. Explosion characteristics, such as lower flammability limit, laminar flame speed, and maximum over-pressure have to be explained by physical principles as pointed out above.

In order to investigate the thermal failure propagation behaviors of Li-ion batteries, full-scale burning tests have to be conducted [21]. Theoretical physical principles have to be worked out on promoting fire safety design of large Li-ion battery energy storage equipment.

\section{Suggestions}

We mention above that heating problem is an important difficulty to overcome in the battery technology. So let us see the reasons behind the heating:

It is not due to the current flow in the battery. It is due to the high wattage, and the current it draws from the battery that must go through the battery con- 
nection, and the inter layer possible voltage gradient due to the difference in the voltage caused by the atomic orbital of non-perfect $2 \mathrm{D}$ structure of the total $\mathrm{N}$ parallel 2D Lithium unit cell grouped in separated cross sections and the number of $n$ layers, that gives the battery voltage $V=n G$ as in Figure 2 .

In many current $\mathrm{Li}$-ion batteries, $\mathrm{Li}$ metal pieces are randomly mixed into $\mathrm{CoO}_{2}$ [1] as in Figure 2(a). $\mathrm{CoO}_{2}$ is an insulator, under its melting temperature. While as the battery energy is drawn, heat is generated if it contacts are normal conductors, causing short circuit and danger of fire or even exploding.

Both these problems can be eliminated if the material separating the layers and the connecting terminals are superconducting, as the superconducting separation between layers will equalize the voltage of all the layers, and will remove the connection heating as well.

High critical temperature (HTC) ceramics in its normal phase is a hole conductor, and as current is drawn, the Li-ions battery will lose its voltage as well as generate heat in the ceramic. However, if the HTC medium is in the superconducting phase as in Figure 2(b), the Li-ion will not lose energy. Such a Li-ion battery must then be housed within a temperature enclosure below the Tc of the HTC ceramic. In another word, such a battery design is only useful for use in very power intensive devices, such as electric vehicles.

When current is passed through a metal wire connected to an HTC superconductor, where the charge carriers are VB holes, due to an applied voltage, say from connecting to a Li-ion battery, it does not lose energy and due also to the effective mass difference; the electrons in the metal wire cannot fill the HTC holes. In fact, due to momentum conservation, it can only have elastic scattering and maintains current continuity. Meaning we can prevent a quick sudden heating as the Li-ion battery is connected to a superconductor instead of a normal conductor as current is drawn. There is of course certain minor loss in energy at the joint. Hopefully it can be dissipated by thermal conduction.

But a superconductor only works in a temperature bath below its critical

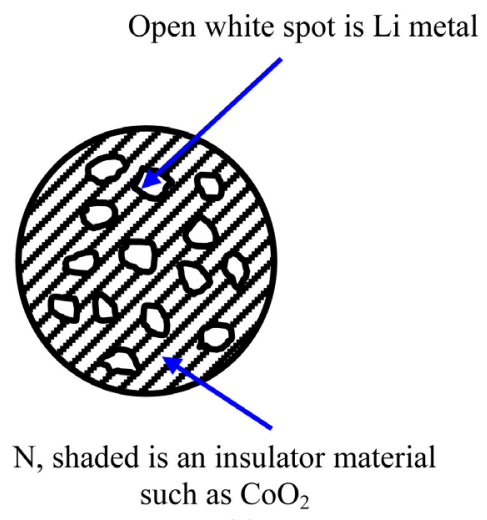

(a)

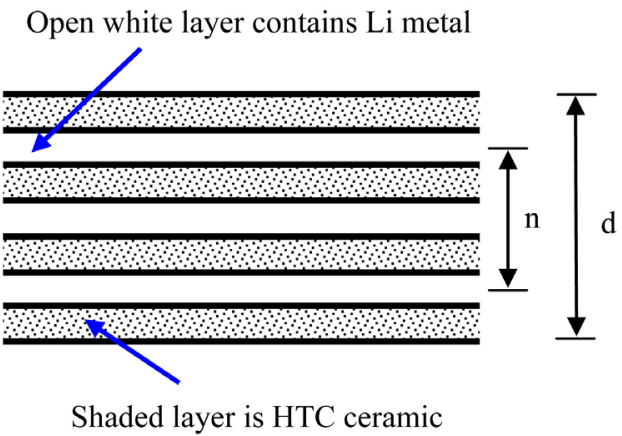

(b)

Figure 2. Grouped Li cells unit. It is vital that the Li layer is made of insulator material to prevent internal short circuit of the battery. (a) Li with insulation materials; (b) Li metal layers with HTC layers separation. 
temperature Tc. Thus the higher the material Tc, the less cooling requirement would be needed. At the present, our best material is the HTC ceramics, such as $\mathrm{YBCO} / \mathrm{BiSCO}$. For these materials, we only need to maintain the battery in liquid nitrogen. With the above reasoning, we propose the battery manufacturing by using layer by layer disposition of Li micro particles with HTC powder, and heated under pressure. Actually, we had previously achieved a high current laser deposition of gold added TBCCO thin film on LaAlO substrate, which can be modified for the Li battery making [22]. First replace the substrate with an HTC ceramic. Then laser deposit a Li film, with the $\mathrm{N}$ cross section geometry, followed by a slightly thicker HTC film, and then the next Li film, etc., until it reaches n Li layers, and lastly sealed with a thick HTC top layer.

In order to improve both the Li-ion battery's voltage and its wattage power, there are two technical methods that one can implement in its design. In increasing the voltage, we must increase the VB binding. The space dimension restriction of the Lithium metal structure to as close as possible to $2 \mathrm{D}$ is achievable in applying nano deposition technology, while the increases in the battery wattage can be achieved by increasing the number of multiple separated layers of the Lithium metal. At present such techniques are limited by the potential current that is generated between the layers, that would generate internal heating, if the separation material between the Lithium layers is made of non-superconducting matter. Our suggested design of using HTC and keeping the battery at liquid nitrogen temperature is new.

Mining Lithium metal is energy intensive and mainly from South America and Australia. Thus it also contributes to environmental problem, despite the clean environment nature of using it as a battery. However, used and discarded Li-ion batteries can be recycled as the Lithium metal in it can be extracted and reused, without new supply from mining. Therefore, for environmental concern, we need to consider this recycling in mind as we design new batteries.

\section{Conclusions}

Basic physics behind Li-ion batteries has been explained in simple terms. Heating problem that will give fire and explosion as experienced has also been alerted to. Suggestions to solve the heating problem have also been proposed.

As we mentioned throughout this paper, the future of electrical power devices, including but not limited to electrical vehicles is the usage of the Lithium-ion battery. But current such batteries suffer from limitation on its voltage, and wattage due much by the internal heating problem that must be overcome. Our suggested new design is totally achievable from current HTC ceramics and laser deposition technologies, plus the thermal cycle cooling chamber technology in keeping the battery at liquid nitrogen temperature and thus minimizing that potential of battery fire danger during recharging and usage. Due to the carbon emission in current energy generators causing climate change, it is paramount that such carbon-based energy devices be eliminated completely within the next couple of decades. For which, the broad use of the Li-ion battery is a key com- 
ponent.

\section{Funding}

The work described in this paper was supported by a grant from the Research Grants Council of the Hong Kong Special Administrative Region for the Theme-Based Research Scheme Project "Safety, Reliability, and Disruption Management of High Speed Rail and Metro Systems" (T32-101/15-R) with account number3-RBAC.

\section{Acknowledgements}

We thank Ms Winnie So for her careful help in editing.

\section{Conflicts of Interest}

The authors declare no conflicts of interest regarding the publication of this paper.

\section{References}

[1] Mizushima, K., Jones, P.C., Wiseman, P.J. and Goodenough, J.B. (1980) Materials Research Bulletin, 15, 783-789. https://doi.org/10.1016/0025-5408(80)90012-4

[2] Mizushima, K., Jones, P.C., Wiseman, P.J. and Goodenough, J.B. (1981) Solid State Ionics, 3, 171-174. https://doi.org/10.1016/0167-2738(81)90077-1

[3] Scrosati, B. and Garche, J. (2010) Journal of Power Sources, 195, 2419-2430. https://doi.org/10.1016/j.jpowsour.2009.11.048

[4] Etacheri, V., Marom, R., Elazari, R., Salitra, G. and Aurbach, D. (2011) Energy and Environmental Science, 4, 3243-3262. https://doi.org/10.1039/c1ee01598b

[5] Yan, L.M., Su, J.M., Sun, C. and Yue, B.H. (2014) Advances in Manufacturing, 2, 358-368. https://doi.org/10.1007/s40436-014-0086-X

[6] Xu, J., Lin, F., Doeff, M.M. and Tong, W. (2017) Journal of Materials Chemistry, 5, 874-901. https://doi.org/10.1039/C6TA07991A

[7] Ali, M.U., Zafar, A., Nengroo, S.H., Hussain, S., Alvi, M.J. and Kim, H.J. (2019) Energies, 12, 446. https://doi.org/10.3390/en12030446

[8] Stampatori, D., Raimondi, P.P. and Noussan, M. (2020) Energies, 13, 2638. https://doi.org/10.3390/en13102638

[9] Lisbona, D. and Snee, T. (2011) Process Safety and Environmental Protection, 89, 434-442. https://doi.org/10.1016/j.psep.2011.06.022

[10] Zhang, Z.J., Ramadass, P. and Fang, W. (2013) Safety of Lithium-Ion Batteries. In: Pistoia, G., Ed., Lithium-Ion Batteries Advances and Applications, Elsevier, Amsterdam, Chapter 18, 409. https://doi.org/10.1016/B978-0-444-59513-3.00018-2

[11] Wu, M.S., Xu, B. and Ouyang, C.Y. (2016) Chinese Physics B, 25, Article ID: 018206. https://doi.org/10.1088/1674-1056/25/1/018206

[12] Gao, J., Shi, S.-Q. and Li, H. (2016) Chinese Physics B, 25, Article ID: 018210. https://doi.org/10.1088/1674-1056/25/1/018210

[13] Wong, K.W. and Chow, W.K. (2020) Journal of Modern Physics, 11, 1058-1074. https://doi.org/10.4236/jmp.2020.117067

[14] Wikipedia, Bohr Model. https://en.wikipedia.org/wiki/Bohr_model\#: :text=In\%20atomic\%20physics\%2C\%2 
0the $\% 20$ Bohr,forces\%20in\%20place $\% 20$ of $\% 20$ gravity

[15] San Jose State University. A Generalization of the Bohr Equation for the Ionization Energy of the Outer Electron of Hydrogen-Like Atoms.

https://www.sjsu.edu/faculty/watkins/bohrgen.htm

[16] Baird, A.R., Archibald, E.J., Marr, K.C. and Ezekoye, O.A. (2020) Journal of Power Sources, 446, Article ID: 227257. https://doi.org/10.1016/j.jpowsour.2019.227257

[17] Johnson, S.K. (2020) Tesla Research Partnership Progresses on New Battery Chemistry, 14 August 2020.

https://arstechnica.com/science/2020/08/tesla-research-partnership-progresses-onnew-battery-chemistry

[18] IEEE 1625:2008, IEEE Standard for Rechargeable Batteries for Multi-Cell Mobile Computing Devices.

[19] Kwok, J.H.Y., Cheng, C.H., Chow, W.K. and Chow, C.L. (2019) An Experimental Study on Possible Thermal Hazards of Cellular Phones. Proceedings of the Third International Fire Safety Symposium (IFireSS 2019), Ottawa, 5-7 June 2019, 597-606.

[20] Luk, S.Y. and Chow, W.K. (2020) Potential Thermal Fire Hazard of Smart Phones and Tablets. Article Submitted to Energy and Environment for Consideration to Publish.

[21] Chen, M.Y., Liu, J.H., Ouyang, D.X., Weng, J.W., Wu, X.Y., Cao, S.C. and Wang, J. (2020) Journal of Energy Storage, 31, Article ID: 101657.

https://doi.org/10.1016/j.est.2020.101657

[22] Wong, K.W., et al. (1994) Laser in Engineering, 2, 319-324. 